\title{
Prognostic Significance of Preterm Isolated Decreased Fetal Movement
}

\author{
Ertuğrul KARAHANOĞLU ${ }^{1}$, Orhan ALTINBOĞA ${ }^{1}$, Funda AKPINAR ${ }^{1}$, Şafak ÖZDEMİRCì' ${ }^{1}$ Emre BASER ${ }^{1}$ \\ Yıldız AKTAŞ', Serdar YALVAÇ¹
}

Ankara, Turkey

\section{ABSTRACT}

OBJECTIVE: Our aim is to evaluate the prognostic significance of isolated, preterm decreased fetal movement following normal initial full diagnostic workup.

STUDY DESIGN: A retrospective observational study was conducted at a tertiary centre. The applied protocol was approved by the Medical Research Ethics Department of the hospital where the research was conducted. Obstetrics outcomes of preterm- and term-decreased fetal movement were compared following an initial, normal diagnostic work up. Evaluated outcomes were birth weight, mode of delivery, stillbirth rate, induction of labour, development of gestational hypertension, small for gestational age and oligohydramnios, polyhydramnios during the follow up period.

RESULT: Obstetric complications related to placental insufficiency develops more frequently for decreased fetal movement in preterm cases with respect to that of in term cases. Following the diagnosis of decreased fetal movement, pregnancy hypertension occurred in $17 \%$ of preterm decreased fetal movement cases and in $4.7 \%$ of term decreased fetal movement cases. Fetal growth restriction developed in $6.6 \%$ of preterm decreased fetal movement and in $2.3 \%$ of term decreased fetal movement. Amniotic fluid abnormalities more frequently developed in preterm decreased fetal movement.

CONCLUSION: Following an initial normal diagnostic workup, preterm decreased fetal movement convey a higher risk for the development of pregnancy complications associated with placental insufficiency. The patient should be monitored closely and management protocols must be developed for initial normal diagnostic workups in cases of preterm decreased fetal movement.

Keywords: Decreased fetal movement, Outcomes of pregnancy, Preterm

Gynecol Obstet Reprod Med 2017;23(2):129-132

\section{Introduction}

Decreased fetal movement (DFM) is an important aspect of antenatal care (1). Between $5 \%$ and $16 \%$ of pregnant women are admitted to their healthcare provider for perception of DFM $(1,2)$. Perceived decreased fetal movement prior to delivery is associated with abnormal blood arterial gas val-



\begin{tabular}{|c|c|}
\hline \multicolumn{2}{|c|}{ Access this article online } \\
\hline $\begin{array}{c}\text { Quick Response Code: } \\
\text { 口 }\end{array}$ & Website: www.gorm.com.tr \\
\hline 好突: & DOI:10.201613/GORM.2016.674 \\
\hline
\end{tabular}

How to cite this article: Karahanoğlu E. Altınboğa O. Akpınar F. Özdemirci S. Baser E. Aktas Y. Yalvaç S. Prognostic Significance of Preterm Isolated Decreased Fetal Movement. Gynecol Obstet Reprod Med 2017;23(3):129132 ues and abnormal placental pathology, which is suggestive of placental insufficiency (3-5). Moreover, decreased fetal movement during pregnancy is associated with adverse pregnancy outcomes such as SGA (small for gestational age), preterm birth related to placental insufficiency and intra-uterine fetal death $(6,7)$. The primary aim of integrating DFM with antenatal care is to decrease stillbirth rates (1). Prospective studies have shown that a planned healthcare package for decreased fetal movement can help to reduce the still birth rate (1).

Accepted definitions for DFM include: (1) the maternal perception of subjective decreased fetal movement; (2) a minimum of 10 movements over two hours, or within 10 hours a minimum of 10 movements (8-10). No method for the evaluation of decreased fetal movement is superior to another. Although no consensus exists about the most reliable method for the diagnosis of DFM, the generally accepted protocol is the mother's own perception of decreased fetal movement $(8,11,12)$.

Following the detection of decreased fetal movement, the management protocol is ultrasonographic evaluation of fetal biometry, fetal cardiotocography and amniotic fluid assessment (13). Prognosis depends on the detected pathologic conditions 
(13). However, in these cohort vast majority aetiology cannot be detected (13). It should also be kept in mind that the sensitivity of ultrasound for the detection of fetal growth restriction is $70 \%$ and fetal biometry do not always reflect placental insufficiency; Fetal decreased movement may reflect fetal compromise prior to emerging the any abnormality detected by conventional diagnostic tools like ultrasound and fetal cardiotocography. There currently exists no consensus regarding how antenatal care must be planned following a diagnosis of decreased fetal movement if no abnormality is detected.

Our aim is to evaluate the prognostic significance of isolated preterm decreased fetal movement following a full diagnostic workup.

\section{Material and Method}

A retrospective observational study was conducted at a tertiary centre. The applied protocol was approved by the Medical Research Ethics Department of the hospital where the research was conducted (Medical Research Ethic Department approval number: 2016-209).

Isolated decreased fetal movement was defined as the maternal perception of decreased fetal movement without any identified aetiology. Following diagnosis of decreased fetal movement, an accepted package for management included fetal biometry, fetal cardiotocography and amniotic fluid assessment. A minimum one-day hospitalization was effected following the DFM.

Management depends on underlying aetiology and pregnancy week at diagnosis. Prior to 39 weeks, if aetiology cannot be defined, the patient should be closely monitored. After 39 weeks of pregnancy, in case of decreased fetal movement, induction of labour was accepted as a part of the protocol.

Evaluated outcomes were birth weight, mode of delivery, stillbirth rate, induction of labour, development of gestational hypertension, SGA and oligohydramnios and polyhydramnios during the follow-up period, following a normal initial evaluation.

Stillbirth was defined as an infant born with no signs of life at or after 24 weeks' gestation. 'Small' for gestational age was defined as an estimated fetal weight lower than the $10^{\text {th }}$ percentile during the follow up period. Oligohydramnios was defined as an amniotic fluid index lower than the $10^{\text {th }}$ percentile prior to 37 weeks of pregnancy and below $50 \mathrm{~mm}$ following 37 weeks of pregnancy (14). Polyhydramnios was defined as an amniotic fluid index (AFI) greater than $24 \mathrm{~cm}$, without any associated fetal or maternal abnormality (14). Hypertension during pregnancy was defined as a measured blood pressure higher than systolic $140 \mathrm{mmHg}$ and diastolic $90 \mathrm{mmHg}$, at 2 occasions and at least six hours apart.

The examinations were performed by five maternal and fetal medicine subspecialists with at least three years of special training in this field. All measurements were taken using Voluson 730 machines (GE Healthcare, Kretz Ultrasound, Zipf, Austria), equipped with convex 4-8 $\mathrm{MHz}$ abdominal transducers. Estimated fetal weight was calculated using the Hadlock formula.

If decreased fetal movement was first identified between 27 to 37 weeks it was defined as preterm decreased fetal movement; if decreased fetal movement was first identified after 37 it was defined as term decreased fetal movement. The evaluated obstetric outcomes were then compared between the two above groups.

The Statistics Package for Social Sciences version 18.0 (SPSS Inc., Chicago, IL, USA) was used for statistical analysis. Kolmogorov-Smirnov and Shapiro-Wilk tests, as well as Q-Q plot graphics, were employed for the assessment of the normal distribution of parameters. Chi-square and Fisher's exact tests were also used in the analysis when required. A ttest for independent samples was used to obtain the numeric values assessment in the form of a parametric test. If the parametric test requirements were not met, a Mann-Whitney U test was used to compare the means between groups. Values were defined as mean \pm standard deviation. The confidence interval was assumed to be $\mathrm{p}<0.05$.

\section{Result}

During the study period, the hospital records of 39480 patients were evaluated. From this cohort, 1895 had a diagnosis of isolated decreased fetal movement and 1114 patients met the inclusion criteria for the study. Incidence of isolated decreased fetal movement was $4.8 \%$. There were 427 preterm decreased fetal movement cases and 687 term decreased fetal movement cases. Patients more frequently perceived reduced fetal movement at term $(61.7 \%)$ than during the preterm pe$\operatorname{riod}(38.3 \%)$.

The demographic characteristics of preterm decreased fetal movement and term decreased fetal movement cases are provided in table 1. Mean maternal age of preterm decreased fetal movement was slightly lower than for term decreased fetal movement. At the preterm period, reference week to health care provider for decreased fetal movement was $33.9 \pm 2.9$, while this was $39.0 \pm 0.96$ at term. Preterm decreased fetal movement patients delivered earlier than their term decreased fetal movement counterparts $(37.8 \pm 1.7$ week vs. $39.5 \pm 0.9$ week).

The obstetrics outcomes of isolated decreased fetal movement are provided in table 2. Caesarean rate was $37.7 \%$ among preterm period patients and 34.2 for term period patients. The need for labour induction was higher among preterm decreased fetal movement patients than for their term decreased fetal movement counterparts $(29.7 \%$ vs. $19.1 \%)$. 
Table 1: Demographic characteristics of groups

\begin{tabular}{lccc}
\hline & $\begin{array}{c}\text { Preterm decreased fetal movement } \\
(\mathrm{n}=417)\end{array}$ & $\begin{array}{c}\text { Term decreased fetal movement } \\
(\mathrm{n}=687)\end{array}$ & $\begin{array}{c}p \\
\text { value }\end{array}$ \\
\hline Age (year) & $26.8 \pm 6.2$ & $26.02 \pm 5.6$ & 0.017 \\
First administration for DFM(week) & $33.9 \pm 2.9$ & $39.0 \pm 0.96$ & 0.00 \\
Delivery week & $37.8 \pm 1.7$ & $39.5 \pm 0.9$ & 0.00 \\
\hline
\end{tabular}

(Total $n=1114$ ) DFM, decreased fetal movement

Table 2. Obstetric outcomes of preterm and term isolated decreased fetal movement

\begin{tabular}{lccc}
\hline Variable & $\begin{array}{c}\text { Preterm decreased isolated fetal movement } \\
(\mathrm{n}=427)\end{array}$ & $\begin{array}{c}\text { Term decreased isolated fetal movement } \\
(\mathrm{n}=687)\end{array}$ & $\begin{array}{c}p \\
\text { value }\end{array}$ \\
\hline Cesarean rate (\%) & $161(37.7)$ & $235(34.2)$ & 0.73 \\
Induction of labor(\%) & $127(29.7)$ & $131(19.1)$ & $<0.01$ \\
Pregnancy hypertension(\%) & $62(17.0)$ & $31(4.7)$ & $<0.01$ \\
FGR(\%) & $38(6.6)$ & $16(2.3)$ & $<0.01$ \\
Oligohydramnios (\%) & $63(14.7)$ & $37(5.3)$ & $<0.01$ \\
Polihydramnios (\%) & $43(10.1)$ & $23(3.3)$ & $<0.01$ \\
Intrauterine fetal death (\%) & $2(0.4)$ & $0(0)$ & $<0.01$ \\
Birth weight (gr) & $3156 \pm 527$ & $3404 \pm 412$ & $<0.01$ \\
\hline
\end{tabular}

(Total $n=1114$ )

During the follow up period, from first admission to delivery, patients with preterm decreased fetal movement had a higher rate of obstetric complications than patients with term decreased fetal movement. Following the diagnosis of decreased fetal movement, pregnancy hypertension rate was $17 \%$ among preterm decreased fetal movement patients and $4.7 \%$ among their term decreased fetal movement counterparts. Amniotic fluid volume abnormalities were more frequently detected in preterm decreased fetal movement patients than their term decreased fetal movement counterparts. Development of oligohydramnios was $14.7 \%$ among preterm decreased fetal movement and 5.3\% among their term decreased fetal movement counterparts. During the follow-up period, intra-uterine fetal death rate was higher among preterm decreased fetal movement patients than their term decreased fetal movement. Birth weight among preterm decreased fetal movement patients was lower than for term decreased fetal movement patients (3156 gr versus 3404gr; $\mathrm{p}<0.001$ ).

\section{Discussion}

The primary aim of antenatal care is to decrease perinatal mortality and morbidity by detecting and predicting pregnancy complications. Integrating fetal movement count with antenatal care is aimed at decreasing intra uterine fetal demise by detecting complications associated with decreased fetal movement (1).

Decreased fetal movement is associated with intra-uterine growth restriction and low birth weight $(15,16)$. Many societies have integrated decreased fetal movement count with antenatal care packages (17). Decreased fetal movement during the preterm period can be used as a screening test for adverse pregnancy complications related to placental insufficiency. The debate regarding how to implement decreased fetal movement into antenatal care packages remains, in addition to how it should be management following diagnosis (18). In the case of preterm decreased fetal movement, even if initial evaluation with conventional diagnostic tools does not detect any abnormality (maternal blood pressure, greyscale ultrasound imaging, Doppler imaging, cardiotocography), during the follow up period, there will be a higher risk for the development of hypertensive pregnancy and SGA.

Patients with preterm decreased fetal movement delivered early and birth weight was lower than in cases of term decreased fetal movement. Isolated preterm decreased fetal movement should not exclusively be accepted as a benign condition. In cases of isolated preterm decreased fetal movement, abnormality may nonetheless be present; this abnormality can progresses during the pregnancy and later present itself as pregnancy hypertension, SGA and placental insufficiency. The mother can detect this abnormality earlier than the conventional medical diagnostic tools currently in use (ultrasound, fetal cardiotocography). Actively including the mother in antenatal care and employing her as a screening tool is an inexpensive and easy approach for decreasing perinatal mortality and morbidity associated with placental insufficiency.

Following a complaint of decreased fetal movement and not detecting any abnormality, the patient should be monitored as indicating a high-risk pregnancy that may develop preeclampsia, SGA and placental insufficiency. The patient should be informed about possible complications that may arise. The clinician should keep in mind that a normal initial 
investigation will not necessarily rule out any abnormality presenting during the follow-up period.

Placental insufficiency and its clinical manifestations like preeclampsia and SGA was ruled a dynamic rather than a static process. Placental insufficiency and related pathologies generally begin at the start or at some point during the pregnancy and only presents clinically at a later stage. Many mathematical models have been developed for the prediction of preeclampsia, SGA and other pathological conditions associated with placental insufficiency (19). Biomarkers, ultrasonographic markers and maternal characteristics are used to predict these conditions (20). As decreased fetal movement is associated with adverse pregnancy complications it can be used as a screening test for the prediction of these complications.

Preterm decreased fetal movement differs from term decreased fetal movement. Although the mother may experience decreased fetal movement more frequently during her term, complications develop more frequently during the preterm period and during the follow-up period. Two explanations may suffice for this difference; first time period is shorter in term period to developed any complication; secondly, the nature of preterm decreased fetal movement may be different from term decreased fetal movement. Whenever the patient complains about decreased fetal movement (preterm) it must be seriously considered.

As our study was retrospective in nature, it contains biases such as recall and selection. To evaluate the exact nature and prognosis of isolated preterm decreased fetal movement, more prospective studies are needed.

\section{References}

1. Tveit JV, Saastad E, Stray-Pedersen B, Bordahl PE, Flenady V, Fretts R, et al. Reduction of late stillbirth with the introduction of fetal movement information and guidelines - a clinical quality improvement. BMC Pregnan Childbirth 2009;9:32.

2. Sergent F, Lefevre A, Verspyck E, Marpeau L. Decreased fetal movements in the third trimester: what to do? Gynecol Obstet Fertil 2005;33(11):861-9.

3. Vintzileos AM, Fleming AD, Scorza WE, Wolf EJ, Balducci J, Campbell WA, et al. Relationship between fetal biophysical activities and umbilical cord blood gas values. Am J Obstet Gynecol 1991;165(3):707-13.

4. Warrander LK, Batra G, Bernatavicius G, Greenwood SL, Dutton P, Jones RL, et al. Maternal perception of reduced fetal movements is associated with altered placental structure and function. PloS One 2012;7(4):e34851.

5. Warrander LK, Heazell AE. Identifying placental dysfunction in women with reduced fetal movements can be used to predict patients at increased risk of pregnancy complications. Medical Hypotheses 2011;76(1):17-20.

6. Froen JF, Arnestad M, Frey K, Vege A, Saugstad OD,
Stray-Pedersen B. Risk factors for sudden intrauterine unexplained death: epidemiologic characteristics of singleton cases in Oslo, Norway, 1986-1995. Am J Obstet Gynecol 2001;184(4):694-702.

7. Sadovsky E, Ohel G, Havazeleth H, Steinwell A, Penchas S. The definition and the significance of decreased fetal movements. Acta Obstet Gynecol Scand 1983;62(5):409-13.

8. Froen JF. A kick from within--fetal movement counting and the cancelled progress in antenatal care. J Perinat Med 2004;32(1):13-24.

9. Mangesi L, Hofmeyr GJ. Fetal movement counting for assessment of fetal wellbeing. Cochrane Database Syst Rev 2007(1):CD004909.

10. Olesen AG, Svare JA. Decreased fetal movements: background, assessment, and clinical management. Acta obstetricia et gynecologica Scandinavica 2004;83(9):818-26.

11. Berbey R, Manduley A, Vigil-De Gracia P. Counting fetal movements as a universal test for fetal wellbeing. Int $\mathrm{J}$ Gynaecol Obstet 2001;74(3):293-5.

12. Froen JF, Heazell AE, Tveit JV, Saastad E, Fretts RC, Flenady V. Fetal movement assessment. Semin Perinatol 2008;32(4):243-6.

13. Froen JF, Tveit JV, Saastad E, Bordahl PE, Stray-Pedersen B, Heazell AE, et al. Management of decreased fetal movements. Semin Perinatol 2008;32(4):307-11.

14. Reddy UM, Abuhamad AZ, Levine D, Saade GR, Fetal Imaging Workshop Invited Participants. Obst Gynecol 2014;123(5):1070-82.

15. Holm Tveit JV, Saastad E, Stray-Pedersen B, Bordahl PE, Froen JF. Maternal characteristics and pregnancy outcomes in women presenting with decreased fetal movements in late pregnancy. Acta Obstet Gynecol Scand 2009;88(12):1345-51.

16. Scala C, Bhide A, Familiari A, Pagani G, Khalil A, Papageorghiou A, et al. Number of episodes of reduced fetal movement at term: association with adverse perinatal outcome. Am J Obstet Gynecol 2015;213(5):678 e1-6.

17. Pediatrics AAo, Obstetricians ACo, Gynecologists. Guidelines for perinatal care: Amer Academy of Pediatrics 2002.

18. Hofmeyr GJ, Novikova N. Management of reported decreased fetal movements for improving pregnancy outcomes. Cochrane Database Sys Rev 2012(4):CD009148.

19. O'Gorman N, Wright D, Syngelaki A, Akolekar R, Wright A, Poon LC, et al. Competing risks model in screening for preeclampsia by maternal factors and biomarkers at 11-13 weeks gestation. Am J Obst Gynecol 2016;214(1):103 e1e12.

20. Wright D, Syngelaki A, Akolekar R, Poon LC, Nicolaides $\mathrm{KH}$. Competing risks model in screening for preeclampsia by maternal characteristics and medical history. Am J Obstet Gynecol 2015; 213(1):62 e1-10. 